University of Wollongong

Research Online

Faculty of Engineering - Papers (Archive)

Faculty of Engineering and Information

Sciences

$15-5-1998$

\title{
Nonlinear optical absorption and LO-phonon emission in steady-state terahertz-driven three-dimensional electron gases
}

W. Xu

University of Wollongong

Follow this and additional works at: https://ro.uow.edu.au/engpapers

Part of the Engineering Commons

https://ro.uow.edu.au/engpapers/249

\section{Recommended Citation}

Xu, W.: Nonlinear optical absorption and LO-phonon emission in steady-state terahertz-driven threedimensional electron gases 1998.

https://ro.uow.edu.au/engpapers/249

Research Online is the open access institutional repository for the University of Wollongong. For further information contact the UOW Library: research-pubs@uow.edu.au 


\title{
Nonlinear optical absorption and LO-phonon emission in steady-state terahertz-driven three-dimensional electron gases
}

\author{
W. $\mathrm{Xu}^{*}$ \\ Department of Physics, University of Wollongong, Wollongong, NSW 2522, Australia \\ (Received 30 July 1997; revised manuscript received 24 October 1997)
}

\begin{abstract}
We present a detailed theoretical study on nonlinear transport and optical properties in three-dimensional electron gases (3DEG's) subjected to intense terahertz (THz) electromagnetic radiation fields. The steady-state electronic transition rate induced by electron-photon-phonon interactions in a 3DEG system has been derived by using an approach from which the effects of multiphoton processes can be easily included. By solving the momentum- and energy-balance equations based on a steady-state Boltzmann equation, where electron interactions with LO phonons are taken into account, we have investigated the dependence of electron temperature, relaxation time, mobility, multiphoton absorption and emission, and photon-assisted LO-phonon emission on the intensity and frequency of the THz radiation fields. A number of important and distinctive transport and optical phenomena have been observed and predicted theoretically. These results are pertinent to transport measurements in the presence of a radiation field and to the application of recently developed far-infrared laser sources such as free-electron lasers. [S0163-1829(98)11219-5]
\end{abstract}

\section{INTRODUCTION}

With the development of novel means of scientific investigation, such as free-electron lasers (FEL's), it has become possible to investigate the transport and optical properties of an electronic device under intense far-infrared (FIR) or terahertz $(\mathrm{THz})$ electromagnetic (EM) radiations. FEL's can provide the tunable source of the linearly polarized $\mathrm{THz}$ radiations with which one can study the dependence of the physical properties in a device system on the frequency and intensity of the EM radiation field. THz radiation is of significant impact on the investigation and characterization of condensed-matter materials such as low-dimensional semiconductor systems and nanostructures. Recently, experimental measurements ${ }^{1-5}$ have been conducted in investigating the nonlinear response of a two-dimensional electron gas (2DEG) to $\mathrm{THz}$ radiation fields provided by UCSB (Refs. 1-3) and FELIX (Refs. 4 and 5) FEL's. Some interesting phenomena, such as resonant absorption of $\mathrm{THz}$ radiation, ${ }^{1}$ $\mathrm{THz}$ radiation enhanced electron temperature, ${ }^{2} \mathrm{THz}$-photon induced impact ionization, ${ }^{3}$ LO-phonon bottleneck effect, ${ }^{4}$ THz-photon-assisted resonant tunneling, ${ }^{5}$ etc., were observed in different 2 DEG structures. These experimental observations have impelled further theoretical study. ${ }^{6,7}$ Moreover, the current availability of experimental measurements at $\mathrm{THz}$ EM fields has resulted in proposals for observing photoninduced quantum resonance effects such as magnetophotonphonon resonances. ${ }^{8}$ It can be foreseen that the study of THz-driven electron gases in semiconductor structures will make a major impact in the fields of semiconductor physics and optoelectronics.

At present, most of the experimental and theoretical work on THz-driven electron gases is focused on the 2DEG systems such as GaAs-based heterojunctions and quantum wells. To have a better understanding of the transport and optical phenomena observed in dimensionally reduced electronic systems such as 2DEG's, it is of value to examine how an ideal three-dimensional electron gas (3DEG) responds to such intense $\mathrm{THz}$ EM radiations; this is the main motivation of the present study.

The investigation of nonlinear transport and optical properties in polar semiconductors such as GaAs has been of fundamental importance both for basic physics and for its applications in electronic and optical devices. There exists a number of theoretical studies on high-frequency ac response of bulk semiconductor materials. Important contributions have been made by some authors. ${ }^{9-14}$ However, these investigations focused either on the linear response, ${ }^{9,10}$ or on the situation with a weak ac field under a strong dc bias, ${ }^{11,12}$ or on the problems of the $v-E_{\mathrm{dc}}$ relation ${ }^{13}$ and thermal-noise temperature. ${ }^{14}$ Furthermore, in most theoretical studies ${ }^{9-14}$ on linear and nonlinear ac responses in semiconductors, one often takes the high-frequency (HF) approximation (i.e., based on the diagrams that contribute to the HF conductivity, as shown in Ref. 15) under which (i) only one photon process is taken into consideration; (ii) the effect of the EM radiation field is represented by an ac electric field; (iii) the presence of the ac electric field does not vary the basic features of fundamental quantities such as the electron densitydensity (d-d) correlation function, Green's function, memory function, etc.; and (iv) the influence of the radiation field on the physical properties of the device system is achieved mainly through introducing an effective frequency $\Omega \rightarrow \Omega$ $\pm \omega$, with $\omega$ being the photon frequency, into these functions (especially the Green's function) obtained in the absence of the radiation. Very recently, some authors ${ }^{16,17}$ have attempted to develop theories going beyond the HF treatment. Most importantly, it was found ${ }^{16}$ that when the effects of the EM radiation field are considered more exactly using, e.g., the approach of the gauge-invariant spectral function or of two-time Green's function derived directly from the solution of the time-dependent Schrödinger equation, the Green's function (or electron density of states) of the electronic system may differ sharply from that obtained in the absence of the EM field. Important radiation effects such as the 
blue shift of the absorption edge, arisen from the dynamic Franz-Keldysh effect, have been predicted. This implies that the usage of theories ${ }^{9-15}$ based on the HF approximation (where the Green's function and/or the electron d-d correlation function were employed in the similar form of those in the absence of the EM field) may be greatly limited, especially in the presence of an EM field with relatively low frequency (LF) and high intensity. A theoretical approach ${ }^{17}$ to study electron transport driven by microwave irradiations, in which the multiphoton processes are taken into account, has been developed. However, the work of Ref. 17 dealt only within a low-temperature limit (i.e., $T \rightarrow 0$ ) where electronimpurity scattering is taken into account for Si-based systems.

The brief review given above shows that more work on THz-driven 3DEG's, in particular for GaAs-based systems subject to intense $\mathrm{THz}$ or FIR radiations, is required. This paper is a contribution in this direction. The present study is organized as follows. In Sec. II, we present an approach to deal with electron-photon-phonon interactions in a 3DEG system. This approach has gone beyond the conventional treatment for electron-photon-phonon interactions in the presence of the radiation field. Using this theory, the electron-photon interactions can be included in a more exact way. In conjunction with the conventional transport measurements from which the response of a 3DEG to the radiation field can be detected and studied, in Sec. III we derive the momentum- and energy-balance equations by introducing the electronic transition rate induced by electron-photonphonon interactions, obtained from this study, into the Boltzmann equation. The momentum- and energy-balance equations can be used as a powerful means in order to study the nonlinear transport and optical properties in an electronic system, through calculating the dependence of such quantities, like electron-energy-loss rate and mobility, on the intensity and frequency of the EM radiation. In Sec. IV, we present a detailed consideration for electron-photon-phonon interactions along with transport and optical properties in a GaAs-based 3DEG structure. The numerical results obtained from this study for THz-driven 3DEG's are presented and discussed in Sec. V. Finally, the present study is summarized in Sec. VI.

\section{ELECTRON-PHOTON-PHONON INTERACTIONS}

In this paper, we consider an electronic system that can be described by a single-electron Hamiltonian: $H(t)=H_{0}(t)$ $+H^{\prime}(t)$, where $H^{\prime}(t)$ can be treated as a perturbation. For an ideal 3DEG system subjected to an EM radiation field polarized along the $x$ axis, the Hamiltonian for a noninteracting electron can be written by

$$
H_{0}(t)=\frac{\left[p_{x}-e A(t)\right]^{2}+p_{y}^{2}+p_{z}^{2}}{2 m^{*}} .
$$

Here, (1) a parabolic-conduction band structure has been included; (2) $p_{x}=-i \hbar \partial / \partial x$ is the momentum operator; (3) $\mathbf{A}(t)$ is the vector potential induced by the EM radiation; and (4) $m^{*}$ is the effective-electron-mass. Furthermore, we have used the Coulomb gauge to describe the EM radiation field, in which the vector potential $\mathbf{A}$ and the scalar potential $\phi$ satisfy $\boldsymbol{\nabla} \cdot \mathbf{A}=0$ and $\phi=0$. These gauge conditions correspond to a situation where the charge density $\rho=0$ and the current density $\mathbf{j}=0$, which are true for the case of noninteracting electrons in the absence of scattering, inhomogeneity, external driving field, etc. After using the dipole approximation for the radiation field and taking $A(t)=A_{0} \sin (\omega t)$, with $\omega$ being the frequency of the EM field, the solution of the time-dependent Schrödinger equation, $i \hbar \partial \psi(\mathbf{R}, t) / \partial t$ $=H_{0}(t) \psi(\mathbf{R}, t)$, is obtained as

$$
\begin{aligned}
|\mathbf{K} ; t\rangle & =\psi_{\mathbf{K}}(\mathbf{R}, t) \\
& =|\mathbf{K} ; 0\rangle e^{-i[E(\mathbf{K})+2 \gamma \hbar \omega] t / \hbar} e^{i r_{0} k_{x}[1-\cos (\omega t)]} e^{i \gamma \sin (2 \omega t)},
\end{aligned}
$$

which has been normalized. Here, $\mathbf{R}=(x, y, z), \quad \mathbf{K}$ $=\left(k_{x}, k_{y}, k_{z}\right)$ is the electron wave vector, $|\mathbf{K} ; 0\rangle=|\mathbf{K}\rangle$ $=e^{i \mathbf{K} \cdot \mathbf{R}}$ is the electron wave function at $t=0, E(\mathbf{K})$ $=\hbar^{2} K^{2} / 2 m^{*}, r_{0}=e E_{0} /\left(m^{*} \omega^{2}\right)$ with $E_{0}$ being the strength of the radiation electric field, $\gamma=\left(e E_{0}\right)^{2} /\left(8 \hbar \omega^{3}\right)$, and $2 \gamma \hbar \omega$ is the energy of the radiation field. We have used the relation $E(t)=\partial A(t) / \partial t=E_{0} \cos (\omega t)$ with $E_{0}=\omega A_{0}$.

In the presence of a perturbation Hamiltonian (induced by, e.g., electronic scattering mechanism), $H^{\prime}(t)=H_{j} e^{i \omega_{j} t}$, where $H_{j}$ is time independent and $\omega_{j}$ is the characteristic frequency for the $j$ th scattering center, we now employ the following notations: (i) $|a\rangle=|\mathbf{K} ; t\rangle$ and $E_{a}=E(\mathbf{K})+2 \gamma \hbar \omega$; (ii) $\omega_{b a}=\left(E_{b}-E_{a}+\hbar \omega_{j}\right) / \hbar$; and (iii) $V_{b a}(t)$ $=\left\langle b\left|H^{\prime}(t)\right| a\right\rangle$. Following the standard theory for timedependent perturbations, ${ }^{18}$ the first-order contribution to the amplitude in the $\left\{H_{0}(t)\right\}$ representation, due to the transition from $|a\rangle$ to $|b\rangle$ induced by $H^{\prime}(t)$, can be calculated by

$$
\begin{aligned}
\left\langle b\left|U^{(1)}\right| a\right\rangle= & \int_{0}^{t} d \tau \frac{V_{b a}(\tau)}{i \hbar} \\
= & \frac{\left\langle\mathbf{K}^{\prime}\left|H_{j}\right| \mathbf{K}\right\rangle}{i \hbar} e^{-i r_{0}\left(k_{x}^{\prime}-k_{x}\right)} \\
& \times \int_{0}^{t} d \tau e^{i \omega_{b a} \tau} e^{i r_{0}\left(k_{x}^{\prime}-k_{x}\right) \cos (\omega \tau)} .
\end{aligned}
$$

Using the identity $e^{i z \cos x}=\Sigma_{m=-\infty}^{\infty} i^{m} J_{m}(z) e^{i m x}$, with $J_{m}(x)$ being a Bessel function, the steady-state transition rate for scattering of an electron from a state $|\mathbf{K}\rangle$ to a state $\left|\mathbf{K}^{\prime}\right\rangle$ is obtained as

$$
\begin{aligned}
W\left(\mathbf{K}^{\prime}, \mathbf{K}\right)= & W_{b a}=\lim _{t \rightarrow+\infty} \frac{\partial}{\partial t}\left|\left\langle b\left|U^{(1)}\right| a\right\rangle\right|^{2} \\
= & \frac{2 \pi}{\hbar}\left|\left\langle\mathbf{K}^{\prime}\left|H_{j}\right| \mathbf{K}\right\rangle\right|^{2} \sum_{m=-\infty}^{\infty} J_{m}^{2}\left[r_{0}\left(k_{x}^{\prime}-k_{x}\right)\right] \\
& \times \delta\left[E\left(\mathbf{K}^{\prime}\right)-E(\mathbf{K})+m \hbar \omega+\hbar \omega_{j}\right] .
\end{aligned}
$$

For electron-phonon interactions, after (i) assuming that the system under study can be separated into the electron of interest and the rest of the crystal, i.e., $|\mathbf{K}, c ; t\rangle=|\mathbf{K} ; t\rangle|c\rangle$ where $|c\rangle$ represents the state of the crystal system; (ii) taking the electron-phonon interaction Hamiltonian to be in the form $H_{j}=V_{\mathbf{Q}}\left(a_{\mathbf{Q}} e^{i \mathbf{Q} \cdot \mathbf{R}}+a_{\mathbf{Q}}^{\dagger} e^{-i \mathbf{Q} \cdot \mathbf{R}}\right)$, where $\mathbf{Q}=\left(q_{x}, q_{y}, q_{z}\right)$ 
is the phonon wave vector, $\left(a_{\mathbf{Q}}^{\dagger}, a_{\mathbf{Q}}\right)$ are the canonical conjugate coordinates of the phonon system, and $V_{\mathbf{Q}}$ is the electron-phonon interaction coefficient; and (iii) taking $\omega_{j}$ $\rightarrow \mp \omega_{Q}$, where the sign $-(+)$ refers to absorption (emission) of a phonon with an energy $\hbar \omega_{Q}$, the steady-state transition rate for electron-photon-phonon interactions in a 3DEG system becomes

$$
\begin{aligned}
W\left(\mathbf{K}^{\prime}, \mathbf{K}\right)= & \frac{2 \pi}{\hbar}\left[\begin{array}{c}
N_{Q} \\
N_{Q}+1
\end{array}\right]\left|V_{\mathbf{Q}}\right|^{2} \delta_{\mathbf{K}^{\prime}, \mathbf{K}}+\mathbf{Q}_{m=-\infty} \sum_{m}^{\infty} J_{m}^{2}\left(r_{0} q_{x}\right) \\
& \times \delta\left[E\left(\mathbf{K}^{\prime}\right)-E(\mathbf{K})-m \hbar \omega \mp \hbar \omega_{Q}\right]
\end{aligned}
$$

where $N_{Q}=\left(e^{\hbar \omega_{Q} / \mathrm{k}_{\mathrm{B}}{ }^{\mathrm{T}}-1}\right)^{-1}$ is the phonon occupation number, and the index $m$ corresponds to the process of $m$-photon absorption (emission) when $m>0(m<0)$. In the presence of the EM radiation, the electrons can interact with the radiation field via the channels of photon emission and absorption. In this paper, we limit ourselves to the situation where the hot-phonon effects such as phonon drift can be neglected. Equation (5) exhibits features distinctive for electronphoton-phonon interactions in a 3DEG structure. We note that in sharp contrast to an isotropic transition rate obtained from using Fermi's golden rule in the absence of the EM field, the presence of the linearly polarized EM radiation results in an anisotropic electronic transition rate characterized by the dependence of $r_{0} q_{x}$ via a term $J_{m}^{2}\left(r_{0} q_{x}\right)$. Equation (5) has also reflected the fact that in the presence of the EM radiations, the electron-phonon scattering (or optical absorption and emission) can be accompanied by electronic transitions through the photon absorption and emission (or phonon scattering).

In the absence of the radiation field, i.e., $E_{0}=0$, Eq. (5) becomes that obtained using the Fermi's golden rule, due to the feature $\lim _{x \rightarrow 0} J_{m}(x)=\delta_{0, m}$. For the case of HF and/or low-intensity radiations, i.e., $r_{0} q_{x} \ll 1$, Eq. (5) can be written in the following form:

$$
\begin{aligned}
W\left(\mathbf{K}^{\prime}, \mathbf{K}\right) \simeq & \frac{2 \pi}{\hbar}\left[\begin{array}{c}
N_{Q} \\
N_{Q}+1
\end{array}\right]\left|V_{\mathbf{Q}}\right|^{2} \delta_{\mathbf{K}^{\prime}, \mathbf{K}+\mathbf{Q}} \\
& \times \sum_{m=0}^{\infty} \frac{1}{(m !)^{2}}\left(\frac{r_{0} q_{x}}{2}\right)^{2 m} \\
& \times \delta\left[E\left(\mathbf{K}^{\prime}\right)-E(\mathbf{K}) \mp m \hbar \omega \mp \hbar \omega_{Q}\right] .
\end{aligned}
$$

Here we have used the relation

$$
\begin{aligned}
J_{m}^{2}(x)= & \sum_{k}(-1)^{k}(x / 2)^{2(m+k)}(2 m+2 k) ! \\
& \times[(2 m+k) !]^{-1}[(m+k) !]^{-2}
\end{aligned}
$$

and taken $k=0$ when $x \ll 1$. Previously, the interactions between electrons and phonons and also those between electrons and photons are treated by separate interaction Hamiltonians. ${ }^{19}$ Using this approach, the net effect of electron-phonon interactions in the presence of multiphoton processes can be calculated by the second- or higher-order perturbation theories, which is very complicated and no simple analytic results are available. The transition rate induced by one-photon absorption process (i.e., $m=1$ ) shown by Eq. (6) is identical to that obtained from using the secondorder perturbation theory. ${ }^{20}$ This implies that the electronphoton interactions can only be treated as a perturbation when the condition $r_{0} q_{x} \ll 1$ is satisfied. From these results, one can find that the theoretical approach used in this study for the case of an ideal 3DEG is much more useful to deal with such problems like multiphoton processes and, therefore, is much better than the previous treatment for electronphoton-phonon interactions. For the case where the timedependent Schrödinger equation, in which the EM field is included, can be solved analytically, it is unnecessary to treat electron-phonon and electron-photon interactions separately.

\section{MOMENTUM- AND ENERGY-BALANCE EQUATIONS}

To set up a tractable method to study nonlinear transport and optical properties of an electronic device has been an outstanding problem. It is well known that the balance equation approach is a powerful tool in studying the nonlinear response of an electron gas to the dc and/or ac fields applied. In this paper, we develop a simple model to investigate the transport and optical properties in THz-driven 3DEG's. We start from a steady-state Boltzmann equation for a 3DEG in the case of degenerate statistics,

$$
\begin{aligned}
\frac{F}{\hbar} \cdot \nabla_{\mathbf{K}} f(\mathbf{K})= & g_{s} \sum_{\mathbf{K}^{\prime}}\left\{f\left(\mathbf{K}^{\prime}\right)[1-f(\mathbf{K})] W\left(\mathbf{K}, \mathbf{K}^{\prime}\right)\right. \\
& \left.-f(\mathbf{K})\left[1-f\left(\mathbf{K}^{\prime}\right)\right] W\left(\mathbf{K}^{\prime}, \mathbf{K}\right)\right\},
\end{aligned}
$$

where $f(\mathbf{K})$ is the steady-state momentum distribution function for an electron in a state $|\mathbf{K}\rangle, g_{s}$ accounts for spin degeneracy, and $W\left(\mathbf{K}^{\prime}, \mathbf{K}\right)$ is the steady-state transition rate for an electron scattered from state $|\mathbf{K}\rangle$ to state $\left|\mathbf{K}^{\prime}\right\rangle$. Furthermore, $\mathbf{F}$ is the external force acting on the electron. It should be noted that when the transition probability is calculated from the solution of the Schrödinger equation in which the effects of the EM field have been included, the force term induced by the EM field will not appear in the drift term on the left-hand side of the Boltzmann equation.

In the present study, we consider the situation where the transport and optical properties of a device system are detected and studied by the classical transport measurements. In these experiments, two quantities, the mobility (or conductivity) and the electron-energy-loss rate (EELR), are connected to the optical absorption and phonon emission. When the radiation is polarized along the $x$ direction, one can apply the external dc probing electric fields (or currents) along the $x$ and $y$ direction and measure the current (or field) along the different directions. In this case, one has $\mathbf{F}=-e\left(E_{x}, E_{y}, 0\right)$. Now, we can derive the balance equations from the Boltzmann equation given by Eq. (7). With the balance equation approach, one may detour the difficulties in solving the Boltzmann equation directly and keep the main merits of the Boltzmann equation to describe the response of electrons to the applied fields in the presence of different scattering mechanisms. We have successfully applied this approach to the problem of hot-electron transport in 2DEG's in strong dc and ac fields. ${ }^{7,21}$ Using the balance equation approach based on the Boltzmann equation, the idea is to choose a certain functional form for the electron distribution function (EDF), of which the drifted EDF is popularly used. We assume that 
the electron momentum distribution function can be described by a statistical energy distribution function through $f(\mathbf{K})=f(\mathcal{E}), \quad$ where $\mathcal{E}=\hbar^{2}\left(k_{x}-m^{*} v_{x} / \hbar\right)^{2} / 2 m^{*}+\hbar^{2}\left(k_{y}\right.$ $\left.-m^{*} v_{y} / \hbar\right)^{2} / 2 m^{*}+\hbar^{2} k_{z}^{2} / 2 m^{*}$ and the EDF is drifted by the average electron velocities $\left(v_{x}\right.$ and $\left.v_{y}\right)$ due to the presence of the dc electric fields $\left(E_{x}\right.$ and $\left.E_{y}\right)$. Thus, we can derive the following relation:

$$
\frac{\partial f(\mathbf{K})}{\partial k_{j}}=\frac{\hbar^{2}}{m^{*}}\left(k_{j}-\frac{m^{*}}{\hbar} v_{j}\right) \frac{\partial f(\mathcal{E})}{\partial \mathcal{E}},
$$

which leads to

$$
\begin{gathered}
\sum_{\mathbf{K}} \frac{\partial f(\mathbf{K})}{\partial k_{j}}=0, \\
\sum_{\mathbf{K}} k_{i} \frac{\partial f(\mathbf{K})}{\partial k_{j}}=-\frac{N_{e}}{2} \delta_{i j},
\end{gathered}
$$

with $N_{e}$ being the volume electron density in the system, and

$$
\sum_{\mathbf{K}} E(\mathbf{K}) \frac{\partial f(\mathbf{K})}{\partial k_{j}}=-\frac{\hbar}{2} N_{e} v_{j}
$$

Here we have used the condition of electron-number conservation. After using the density of states for a 3DEG [i.e., $D(E)=\left(2 \pi^{2}\right)^{-1}\left(2 m^{*} / \hbar^{2}\right)^{3 / 2} \sqrt{E} \Theta(E)$, where $E$ is the electron energy], we have

$$
N_{e}=\frac{1}{2 \pi^{2}}\left(\frac{2 m^{*}}{\hbar^{2}}\right)^{3 / 2} \int_{0}^{\infty} d E \sqrt{E} f(E)
$$

where $f(E)$ is the electron-energy-distribution function.

From Eq. (9a), one can find that the mass-balance equation (multiplying $\Sigma_{\mathbf{K}}$ to both sides of the Boltzmann equation) has been balanced by the EDF chosen above. At the first moment, the momentum-balance equations can be derived by multiplying $\Sigma_{\mathbf{K}} k_{j}$ to both sides of the Boltzmann equation. Using Eq. (9b), we have

$$
e E_{j}=\frac{2 q_{s} \hbar}{N_{e}} \sum_{\mathbf{K}^{\prime}, \mathbf{K}}\left(k_{j}^{\prime}-k_{j}\right) f(\mathbf{K})\left[1-f\left(\mathbf{K}^{\prime}\right)\right] W\left(\mathbf{K}^{\prime}, \mathbf{K}\right) .
$$

At the second moment, the energy-balance equation can be derived by multiplying $\Sigma_{\mathbf{K}} E(\mathbf{K})$ to both sides of the Boltzmann equation. After using Eq. (9c), we have

$$
\begin{aligned}
\frac{N_{e} e}{2}\left(E_{x} v_{x}+E_{y} v_{y}\right)= & g_{s} \sum_{\mathbf{K}^{\prime}, \mathbf{K}}\left[E\left(\mathbf{K}^{\prime}\right)-E(\mathbf{K})\right] \\
& \times f(\mathbf{K})\left[1-f\left(\mathbf{K}^{\prime}\right)\right] W\left(\mathbf{K}^{\prime}, \mathbf{K}\right) .
\end{aligned}
$$

Applying free-electron lasers as the driving force to an electron gas, the strength of the radiation field can be very strong and $E_{0}$ can be on the order of $\mathrm{kV} / \mathrm{cm}$. Normally, in the transport experiments the dc probing electric field can be applied on the order of $\mathrm{V} / \mathrm{cm} .{ }^{1,2}$ When the probing dc electric fields are small enough, i.e., $E_{x} \ll 1$ and $E_{y} \ll 1$, the average electron velocities $\left(v_{x}\right.$ and $\left.v_{y}\right)$ are also very small. Expanding $f(\mathbf{K})$ with respect to $v_{j}$ gives

$$
f(\mathbf{K}) \simeq f(X)-\hbar\left(v_{x} k_{x}+v_{y} k_{y}\right) \frac{\partial f(X)}{\partial X},
$$

where $X=\hbar^{2} K^{2} / 2 m^{*}$. When the expansion is terminated with the linear terms of the velocities, the usage of the approximation given by Eq. (13) is equivalent to the relaxation time approximation in the linear response theory. ${ }^{22}$ It should be noted that the linear response discussed here relates only to the probing dc fields applied. Furthermore, at a steady state (in which the effects of the radiation field have been included within the electronic transition rate) and using the momentum- and energy-balance equation approach, we do not need to take a time average in the Boltzmann equation.

After using the approximation given by Eq. (13) and noting that the mobility and conductivity are defined, respectively, by $\mu_{i j}=-v_{j} / E_{i}$ and $\sigma_{i j}=N_{e} e \mu_{i j}$, which can be obtained experimentally from applying the probing electric field along the $i$ direction and measuring the current along the $j$ direction, from the momentum-balance equations we have

$$
\mu_{x y}=\mu_{y x}=0 \quad \text { and } \quad \mu_{j j}=\frac{e}{m^{*}} \tau_{j},
$$

where the momentum-relaxation time in different geometries can be calculated by

$$
\frac{1}{\tau_{j}} \simeq \frac{2 g_{s} \hbar^{2}}{m^{*} N_{e}} \sum_{\mathbf{K}^{\prime}, \mathbf{K}}\left(k_{j}^{\prime}-k_{j}\right) k_{j} \frac{\partial f(X)}{\partial X} W\left(\mathbf{K}^{\prime}, \mathbf{K}\right) .
$$

Using Eq. (13) and noting that the EELR is defined by $P$ $=\left(\sigma_{x x} E_{x}^{2}+\sigma_{y y} E_{y}^{2}\right) / 2$ when $\sigma_{x y}=\sigma_{y x}=0$, from the energybalance equation we have

$$
P \simeq g_{s} \sum_{\mathbf{K}^{\prime}, \mathbf{K}}\left[E(\mathbf{K})-E\left(\mathbf{K}^{\prime}\right)\right] f(X)\left[1-f\left(X^{\prime}\right)\right] W\left(\mathbf{K}^{\prime}, \mathbf{K}\right),
$$

where $X^{\prime}=\hbar^{2} K^{\prime 2} / 2 m^{*}$.

Introducing the steady-state transition rate induced by electron-photon-phonon interactions, i.e., Eq. (5), into Eqs. (15) and (16) and taking $g_{s}=2$, we obtain

$$
\begin{aligned}
\frac{1}{\tau_{j}}= & \frac{m^{* 2}}{4 \pi^{4} \hbar^{5} N_{e}} \sum_{m} \int d^{3} \mathbf{Q}\left[\begin{array}{c}
N_{Q} \\
N_{Q}+1
\end{array}\right] \\
& \times\left|V_{\mathbf{Q}}\right|^{2} J_{m}^{2}\left(r_{0} q_{x}\right) \frac{q_{j}^{2}}{Q^{3}}\left(\varepsilon_{Q}-a_{m}^{ \pm}\right) f(y)
\end{aligned}
$$

and

$$
\begin{aligned}
P= & \frac{-m^{* 2}}{8 \pi^{4} \hbar^{5}} \sum_{m} \int d^{3} \mathbf{Q}\left[\begin{array}{c}
N_{Q} \\
N_{Q}+1
\end{array}\right] \frac{a_{m}^{ \pm}}{Q} \\
& \times\left|V_{Q}\right|^{2} J_{m}^{2}\left(r_{0} q_{x}\right) \int_{0}^{\infty} d x f(x+y)\left[1-f\left(x+y+a_{m}^{ \pm}\right)\right] .
\end{aligned}
$$

Here, $\quad a_{m}^{ \pm}=m \hbar \omega \pm \hbar \omega_{Q}, \quad \varepsilon_{Q}=\hbar^{2} Q^{2} / 2 m *$, and $y=\left(\varepsilon_{Q}\right.$ $\left.-a_{m}^{ \pm}\right)^{2} / 4 \varepsilon_{Q}$. It should be noted that when the effects of the radiation have been included within the electronic transition 
rate, at steady state, the mobility or conductivity will no longer be in the form of the Drude formula ${ }^{7,19}$ through $\sigma$ $=\left(N_{e} e^{2} / m^{*}\right)[\tau /(1+i \omega \tau)]$.

For an electronic system with relatively low electron density and at relatively high temperature (or high electron temperature), the Maxwellian is the most popularly used statistical electron-energy-distribution function. In this paper, we take a Maxwellian-type of electron distribution, characterized by an electron temperature $T_{e}$, as the EDF through

$$
f(x)=c e^{-\beta x},
$$

where $\beta=1 / k_{B} T_{e}$ and $c$ is a normalization factor. Introducing Eq. (19) into the condition of electron number conservation, Eq. (10), one has $c=4 N_{e}\left(\pi \beta \hbar^{2} / 2 m^{*}\right)^{3 / 2}$. Using Eq. (19), the momentum-relaxation time in different geometries becomes

$$
\begin{aligned}
\frac{1}{\tau_{j}}= & \sqrt{2 m^{*} / \pi} \frac{\beta^{3 / 2}}{4 \pi^{2} \hbar^{2}} \sum_{m} \int d^{3} \mathbf{Q}\left[\begin{array}{c}
N_{Q} \\
N_{Q}+1
\end{array}\right]\left|V_{Q}\right|^{2} J_{m}^{2}\left(r_{0} q_{x}\right) \\
& \times \frac{q_{j}^{2}}{Q^{3}}\left(\varepsilon_{Q}-a_{m}^{ \pm}\right) \exp \left[-\frac{\beta}{4} \frac{\left(\varepsilon_{Q}-a_{m}^{ \pm}\right)^{2}}{\varepsilon_{Q}}\right]
\end{aligned}
$$

and the EELR is in the form

$$
\begin{aligned}
P= & -\sqrt{2 m^{*} / \pi} \frac{N_{e} \beta^{1 / 2}}{8 \pi^{2} \hbar^{2}} \sum_{m} \int d^{3} \mathbf{Q}\left[\begin{array}{c}
N_{Q} \\
N_{Q}+1
\end{array}\right] \frac{a_{m}^{ \pm}}{Q}\left|V_{\mathbf{Q}}\right|^{2} \\
& \times J_{m}^{2}\left(r_{0} q_{x}\right) \exp \left[-\frac{\beta}{4} \frac{\left(\varepsilon_{Q}-a_{m}^{ \pm}\right)^{2}}{\varepsilon_{Q}}\right] \\
& \times\left(1-\frac{c}{2} e^{-\beta a_{m}^{ \pm}} \exp \left[-\frac{\beta}{4} \frac{\left(\varepsilon_{Q}-a_{m}^{ \pm}\right)^{2}}{\varepsilon_{Q}}\right]\right) .
\end{aligned}
$$

\section{FOR A GaAs-BASED 3DEG STRUCTURE}

For bulk semiconductors such as GaAs, when the $x, y$, and $z$ directions are chosen along the crystal axes, the electrons in the conduction band (especially in the $\Gamma$ band) can be treated roughly as a $3 \mathrm{DEG},{ }^{23}$ due to (i) the zinc-blende symmetry of the crystal structure; (ii) the parabolic nature of the band structure (especially around the $\Gamma$ point); and (iii) relatively large energy gaps between conduction and valence bands and between different valleys. Moreover, the photon energy of FIR or THz radiations $(\hbar \omega \sim \mathrm{meV})$ is much less than the energy gaps $\left(E_{g} \sim \mathrm{eV}\right)$ among different bands and valleys in GaAs. Therefore, for a THz-driven 3DEG realized from bulk semiconductors such as GaAs, the effects of interband and intervalley transitions via corresponding optical processes can be neglected when the radiation intensity is not extremely high. Hence, the theoretical model and approaches discussed in Secs. II and III in this paper can be used for GaAs-based 3DEG structure.

When an electron gas is subjected to intense EM radiations, the EM field will lead to the excitation of the electrons in the system and to the variation of the electron temperature. For polar semiconductors such as GaAs, the frequency of the phonon oscillation associated with longitudinal-optic (LO) modes is at about $\mathrm{THz}\left(\omega_{\mathrm{LO}} / 2 \pi \simeq 8.85 \mathrm{THz}\right)$. Therefore, the electron-LO-phonon interaction is the principle channel for relaxation of excited electrons in a GaAs-based 3DEG, due to its large energy transfer during a scattering event. The Fröhlich Hamiltonian ${ }^{24}$ can be used to describe the nature of electron-LO-phonon interactions through

$$
V_{\mathbf{Q}}=i\left(4 \pi \alpha L_{0}\right)^{1 / 2} \frac{\hbar \omega_{\mathrm{LO}}}{Q},
$$

where $\hbar \omega_{\mathrm{LO}}$ is the LO-phonon energy at the longwavelength limit, $L_{0}=\left(\hbar / 2 m^{*} \omega_{\mathrm{LO}}\right)^{1 / 2}$ is the polaron radius, and $\alpha$ is the electron-LO-phonon coupling constant. After introducing Eq. (22) into Eqs. (20) and (21) and taking $\omega_{Q}$ $\rightarrow \omega_{\mathrm{LO}}, \quad N_{Q} \rightarrow N_{0}=\left(e^{\hbar \omega_{\mathrm{LO}} / k_{B} T}-1\right)^{-1}, \quad$ and $a_{m}^{ \pm}=m \hbar \omega$ $\pm \hbar \omega_{\mathrm{LO}}$ for LO-phonon scattering, the momentum-relaxation time in different geometries is obtained as

$$
\begin{aligned}
\frac{1}{\tau_{j}}= & \frac{\alpha\left(\beta \hbar \omega_{\mathrm{LO}}\right)^{3 / 2}}{\sqrt{\pi} \hbar}\left[\begin{array}{c}
N_{0} \\
N_{0}+1
\end{array}\right] \sum_{m} \int_{0}^{\infty} \frac{d y}{y}\left(y-a_{m}^{ \pm}\right) \\
& \times \exp \left[-\frac{\beta}{4} \frac{\left(y-a_{m}^{ \pm}\right)^{2}}{y}\right] \\
& \times \int_{0}^{1} d x J_{m}^{2}\left(r_{0} x \sqrt{2 m^{*} y / \hbar^{2}}\right) R_{j}(x),
\end{aligned}
$$

where $R_{x}(z)=2 z^{2}$ and $R_{y}(z)=1-z^{2}$, and the EELR is obtained by

$$
P=P_{\mathrm{LO}}-P_{\mathrm{OP}}
$$

Here, $P_{\text {LO }}$ is the EELR induced by electron-LO-phonon coupling

$$
P_{\mathrm{LO}}=\hbar \omega_{\mathrm{LO}} \sum_{m=-\infty}^{\infty}\left[\left(N_{0}+1\right) I_{m}^{-}-N_{0} I_{m}^{+}\right],
$$

which is the difference between phonon emission and absorption accompanied by different processes of photon emission and absorption. $P_{\mathrm{OP}}\left(-P_{\mathrm{OP}}\right)$ is the EELR (electronenergy-gain rate) induced by electron interactions with the radiation field

$$
P_{\mathrm{OP}}=\sum_{m=-\infty}^{\infty} m \hbar \omega\left[\left(N_{0}+1\right) I_{m}^{-}+N_{0} I_{m}^{+}\right],
$$

which is the summation of the processes caused by emission and absorption of phonons and photons. Furthermore,

$$
\begin{aligned}
I_{m}^{ \pm}= & \alpha \omega_{\mathrm{LO}} N_{e} \sqrt{\beta \hbar \omega_{\mathrm{LO}} / \pi} \int_{0}^{\infty} \frac{d y}{y} \\
& \times \exp \left[-\frac{\beta}{4} \frac{\left(y-a_{m}^{ \pm}\right)^{2}}{y}\right]\left(1-\frac{c}{2} e^{-\beta a_{m}^{ \pm}}\right. \\
& \left.\times \exp \left[-\frac{\beta}{4} \frac{\left(y-a_{m}^{ \pm}\right)^{2}}{y}\right]\right) \\
& \times \int_{0}^{1} d x J_{m}^{2}\left(r_{0} x \sqrt{2 m^{*} y / \hbar^{2}}\right) .
\end{aligned}
$$

From Eq. (24a), we see that for the weak probing electric fields $E_{x} \ll 1$ and $E_{y} \ll 1, P=\left(\sigma_{x x} E_{x}^{2}+\sigma_{y y} E_{y}^{2}\right) / 2 \ll 1$. Taking $P \rightarrow 0$, we have $P_{\mathrm{LO}} \simeq P_{\mathrm{OP}}$, which reflects the fact that the 
electrons in the system gain (or lose) the energy mainly from the EM fields applied via the processes of optical absorption (or emission) and lose (or gain) the energy via the channels for emission (absorption) of phonons. Therefore, for a THzdriven 3DEG, the absorption and emission of phonons (photons) can be achieved by the processes of electronic transitions accompanied by optical (phonon) emission and absorption, which is an indirect photon-absorption (phonon emission) mechanism.

From Eq. (23), one can find that, in the presence of an EM radiation polarized along the $x$ direction, the relaxation time $\tau_{x}$ may be different from that of $\tau_{y}$, because of the anisotropic feature in the electronic transition rate induced electron-photon-phonon interactions. This feature may result in $\mu_{x x} \neq \mu_{y y}$ or $\sigma_{x x} \neq \sigma_{y y}$, which can be measured experimentally. The physical reason behind this anisotropic conduction behavior is that the EM radiation polarized along a certain direction may break the symmetry of the sample geometry.

When $E_{0}=0$ (i.e., $r_{0}=0$ ), due to $J_{m}^{2}(0)=\delta_{0, m}$, we have

$$
\begin{gathered}
\frac{1}{\tau_{x}}=\frac{1}{\tau_{y}}=\frac{1}{\tau}=\omega_{\mathrm{LO}} \frac{4 \alpha \beta_{e}^{3 / 2}}{3 \pi^{1 / 2}} N_{0} e^{\beta_{e} / 2} \\
\times\left[\left(1+e^{\beta_{0}-\beta_{e}}\right) K_{1}\left(\frac{\beta_{e}}{2}\right)+\left(1-e^{\beta_{0}-\beta_{e}}\right) K_{0}\left(\frac{\beta_{e}}{2}\right)\right], \\
P_{\mathrm{LO}}=N_{e} \frac{\hbar \omega_{\mathrm{LO}}}{\tau_{e}},
\end{gathered}
$$

and

$$
\begin{aligned}
\frac{1}{\tau_{e}}= & \omega_{\mathrm{LO}} \frac{2 \alpha \beta_{e}^{1 / 2}}{\pi^{1 / 2}}\left[N _ { 0 } e ^ { \beta _ { e } / 2 } \left(e^{\left.\beta_{0}-\beta_{e}-1\right)}\right.\right. \\
& \left.\times K_{0}\left(\frac{\beta_{e}}{2}\right)-\frac{c}{2} K_{0}\left(\beta_{e}\right)\right] .
\end{aligned}
$$

Here, $\beta_{0}=\hbar \omega_{\mathrm{LO}} / k_{B} T, \beta_{e}=\hbar \omega_{\mathrm{LO}} / k_{B} T_{e}$, and $K_{n}(x)$ is the modified Bessel function. Equations (25) are the well-known results obtained in the absence of the EM radiation. For low electron density electron gas (i.e., $c \ll 1)$ and when $T_{e} \rightarrow T$, $P_{\mathrm{LO}}=0$.

\section{RESULTS AND DISCUSSIONS}

The numerical results of this paper pertain to GaAs-based 3DEG structures. The material parameters for $\mathrm{GaAs}$ are taken as (1) effective-electron-mass ratio $m^{*} / m_{e}=0.0665$ with $m_{e}$ the electron rest mass; (2) electron-LO-phonon coupling constant $\alpha=0.068$; and (3) LO-phonon energy $\hbar \omega_{\mathrm{LO}}$ $=36.6 \mathrm{meV}$ (i.e., $\omega_{\mathrm{LO}} / 2 \pi=8.85 \mathrm{THz}$ ). Furthermore, in all of the calculations, (i) we consider an $n$-type-doped GaAs with the typical electron density $N_{e}=10^{23} \mathrm{~m}^{-3}$; (ii) we take the lattice temperature $T=77 \mathrm{~K}$ at and above which the strong electron-LO-phonon scattering is present in GaAs; and (iii) we include contributions from $m=0, \pm 1, \pm 2, \ldots, \pm 20$ optical channels. Including more multiphoton processes in the calculation influences the results only for low-frequency and high-intensity radiations.

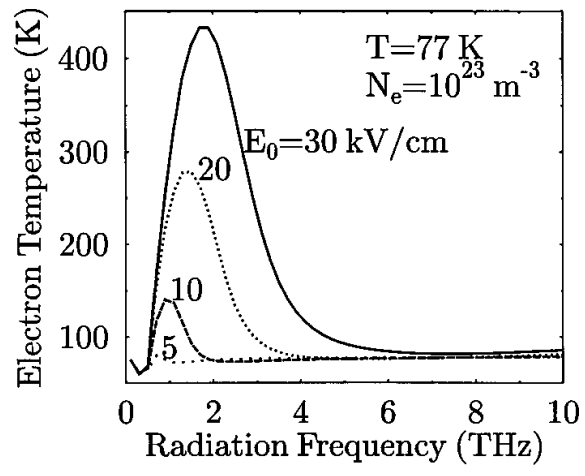

FIG. 1. Electron temperature $T_{e}$ as a function of radiation frequency $\omega / 2 \pi$ for different radiation intensities $E_{0} . \quad N_{e}$ is the electron density.

By solving the energy-balance equation, given by $P_{\mathrm{LO}}$ $=P_{\mathrm{OP}}$ in the case of the weak probing fields, we can obtain the electron temperature $T_{e}$ and the EELR induced by emission and absorption of phonons and photons via different optical processes for a given radiation field with strength $E_{0}$ and frequency $\omega$. Introducing $T_{e}$ obtained into the momentum-balance equations, given by $\mu_{j j}=e \tau_{j} / m^{*}$, we can obtain the mobility $\left(\mu_{x x}\right.$ and $\left.\mu_{y y}\right)$ in different geometries. It is interesting to note that the inclusion of the EM field within the electronic transition rate can simplify considerably the numerical calculation through using the momentum- and energy-balance equation approach. When considering the EM field as an ac driving force in the drift term of the Boltzmann equation, one has to solve selfconsistently the momentum- and energy-balance equations in order to obtain $T_{e} \cdot{ }^{7}$ This is very CPU consuming. Using theoretical approaches developed in this study, we can obtain $T_{e}$ by solving the energy-balance equation alone. Moreover, using these approaches, we can study easily the optical absorption and phonon emission through different channels of multiphoton processes.

\section{A. Electron temperature and electron-energy-loss rate}

The influence of the intensity $E_{0}$ and frequency $\omega$ of the $\mathrm{THz}$ laser radiations on electron temperature of a $3 \mathrm{DEG}$ is shown in Figs. 1 and 2. We find the following. (1) At relatively low-frequency radiations, the electrons in the system will be slightly cooling down, i.e., $T_{e}<T$ (see Fig. 1 and

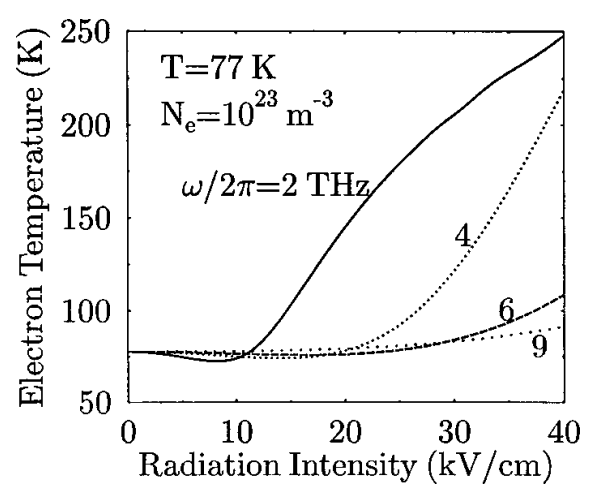

FIG. 2. Electron temperature as a function of radiation intensity for different radiation frequencies. 


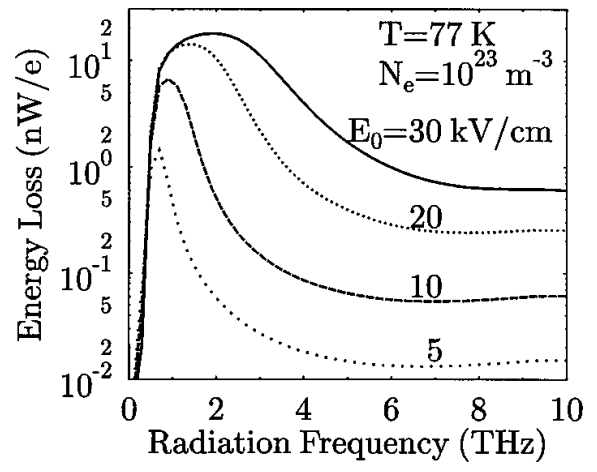

FIG. 3. Total electron-energy-loss rate as a function of radiation frequency $\omega / 2 \pi$ for different radiation intensities $E_{0}$. The results are shown for $P_{\mathrm{LO}} / N_{e}$ or $P_{\mathrm{OP}} / N_{e}$, i.e., the average EELR per electron.

$\omega / 2 \pi=2 \mathrm{THz}$ in Fig. 2). (2) With increasing radiation frequency, $T_{e}$ first increases then decreases (see Fig. 1). The peak of $T_{e}$ shifts to the higher-frequency regime with increasing $E_{0}$. This effect is more pronounced for the highintensity radiations. (3) $T_{e}$ increases with increasing $E_{0}$ (see Fig. 2). The electrons in the system can be more efficiently heated by a relatively LF radiation field. (4) The higher the radiation frequency is, the larger the radiation intensity required to heat the electrons (see Figs. 1 and 2).

Electron temperature measures how efficiently an electron can gain the energy from the fields applied and lose it through the emission of a phonon. In low-intensity and/or low-frequency regimes where $\omega \ll \omega_{\mathrm{LO}}$, the process for an electron to lose the energy via LO-phonon emission is much more efficient than that to gain the energy via photon absorption, due to the larger energy transfer caused by LO-phonon scattering. As a consequence, the requirement $P_{\mathrm{LO}} \simeq P_{\mathrm{OP}}$ may lead to lowering the electron temperature so that the effects of LO-phonon absorption become stronger. This effect results from predominantly forward scattering induced by the LO-phonon scattering mechanism. ${ }^{25}$ The cooling of electrons through this mechanism was observed in nonlinear electron transport in strong dc fields ${ }^{21}$ and in the presence of nonquantizing magnetic fields. ${ }^{25,26}$ At radiations with sufficiently high intensity and high frequency, an electron can be accelerated quickly to reach an energy level $E \geqslant \hbar \omega_{\mathrm{LO}}$. When the time of this process required is shorter than the relaxation time required to emit a LO phonon, electrons will be heated. From Figs. 1 and 2, one can find that for a GaAsbased 3DEG, the heating of electrons occurs at about $\omega / 2 \pi$ $\sim 2 \mathrm{THz}$ when $E_{0}>10 \mathrm{kV} / \mathrm{cm}$. With further increasing radiation frequency so that $\omega \sim \omega_{\mathrm{LO}}$, an electron can easily gain the energy, via the photon absorption, to reach $E \sim \hbar \omega_{\mathrm{LO}}$ and lose the energy via phonon emission. When this two-step process is analogous to an elastic scattering event, the electron gas will not be heated (i.e., $T_{e} \sim T$ ).

The influence of the $\mathrm{THz}$ radiation on the total EELR induced by LO-phonon emission $P_{\mathrm{LO}}$ or photon absorption $P_{\mathrm{OP}}$ (noting $P_{\mathrm{LO}}=P_{\mathrm{OP}}$ ) is shown in Figs. 3 and 4. At a fixed $\omega, T_{e}$ increases with increasing $E_{0}$ so that LO-phonon emission scattering and photon absorption scattering increase with $E_{0}$. As a result, the EELR $\left(P_{\mathrm{LO}}\right.$ or $\left.P_{\mathrm{OP}}\right)$ increases with increasing radiation intensity (see Fig. 4). From Fig. 3, we

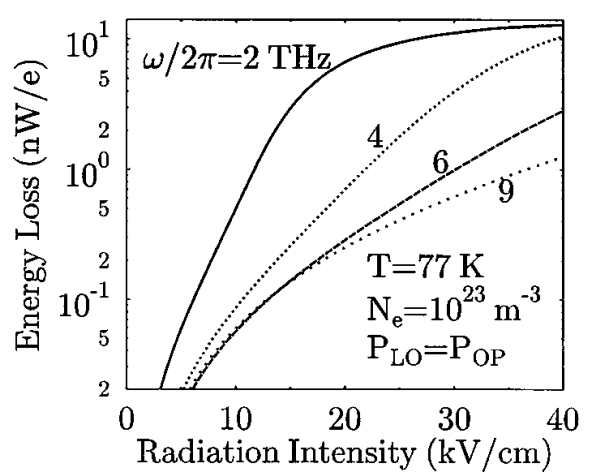

FIG. 4. Total electron-energy-loss rate per electron as a function of radiation intensity for different radiation frequencies.

see that at a fixed radiation intensity, a peak of the optical absorption (or phonon emission) can be observed at about $\omega / 2 \pi \sim 1 \mathrm{THz}$. With increasing $E_{0}$, the peak shifts to the HF regime and looks more broadened. We note that the peak of the optical absorption observed here for a $3 \mathrm{DEG}$ is very similar to the resonant absorption observed in $\mathrm{THz}$-driven 2DEG's (Refs. 1 and 6) where, however, the spectrum of $\mathrm{THz}$ optical absorption was shown at a fixed electron temperature and the peak was observed at about $\omega / 2 \pi$ $\sim 0.5 \mathrm{THz}$.

In the absence of an EM field, the dependence of the EELR induced by LO-phonon scattering on electron temperature can be described by a simple relation: ${ }^{27}$

$$
P_{\mathrm{LO}} / N_{e}=\left(\hbar \omega_{\mathrm{LO}} / \tau^{*}\right) e^{-\hbar \omega_{\mathrm{LO}} / k_{B} T_{e}},
$$

where $\tau^{*}$ is the effective hot-electron relaxation time. Using the data shown in Fig. 4 we can examine the validity of this relation in the presence of the EM radiations, by plotting $\log _{10}\left(P_{\mathrm{LO}} / N_{e}\right)$ versus $1 / T_{e}$, as shown in Fig. 5 for different radiation frequencies. The results shown here indicate that $P_{\mathrm{LO}} \sim e^{-\hbar \omega_{\mathrm{LO}} / k_{B} T_{e}}$ can be roughly seen at high-intensity (or large $T_{e}$ ) radiations. A significant conclusion we draw from Fig. 5 is that in the presence of intense $\mathrm{THz}$ radiations, Eq. (26) can be used to describe the relationship between the EELR and the electron temperature through introducing a frequency-dependent hot-electron relaxation time: $\tau^{*}$ $\rightarrow \tau^{*}(\omega)$. This approach has been employed by Asmar

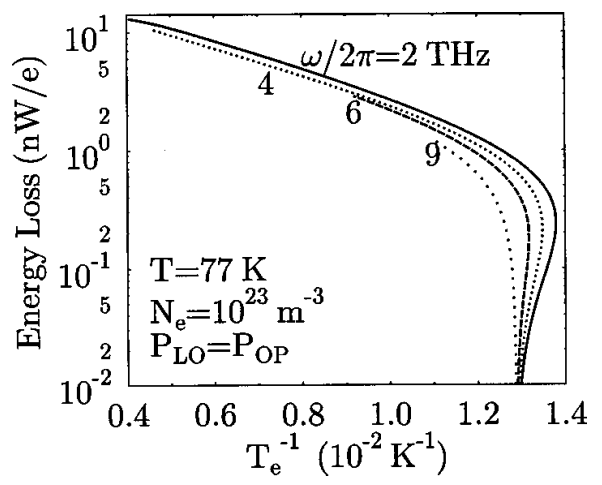

FIG. 5. Total electron-energy-loss rate per electron as a function of the inverse of the electron temperature $T_{e}$ for different radiation frequencies. The results are presented using the data shown in Fig. 4. 


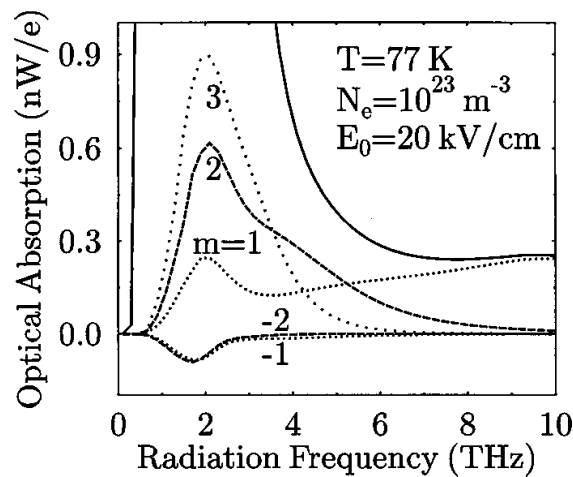

FIG. 6. Optical absorption energy per electron, induced by different photon processes, as a function of radiation frequency $\omega / 2 \pi$ at a fixed radiation intensity. $m>0 \quad(m<0)$ corresponds to a $m$-photon absorption (emission) process and the solid curve is the total contributions from all optical channels (see Fig. 3).

et $a{ }^{1}{ }^{1}$ in analyzing their experimental data. At low-intensity radiations (or small $T_{e}$ ), the dependence of $P_{\mathrm{LO}}$ on $T_{e}$ may be modified strongly by the intensity and frequency of the radiation field.

\section{B. Multiphoton absorption and photon-assisted phonon emission}

The contributions from different optical processes to the total energy of optical absorption in a 3DEG structure are shown in Figs. 6 and 7. As can be seen from Eq. (24c), the process of $m=0$ does not contribute to optical absorption. The optical absorption is an overall contribution to EELR from photon emission $(m<0)$ and absorption $(m>0)$. From Fig. 6, we see that at a fixed radiation intensity, LF optical absorption can be achieved by electronic transitions via multiphoton channels. At $\mathrm{LF}$ radiations $\left(\omega / 2 \pi<2 \mathrm{THz}\right.$ at $E_{0}$ $=20 \mathrm{kV} / \mathrm{cm}$ ), optical absorption via different multiphoton processes increases with $\omega$. With further increasing radiation frequency $(\omega / 2 \pi>2 \mathrm{THz})$, optical absorption via $m \geqslant 2$ channels and via photon emission decreases and the absorption peaks induced by these optical processes can be observed at about $\omega / 2 \pi \sim 2 \mathrm{THz}$. At HF radiations (e.g., $\omega / 2 \pi>6 \mathrm{THz}$ when $E_{0}=20 \mathrm{kV} / \mathrm{cm}$ ), optical absorption is achieved mainly through one-photon absorption process.

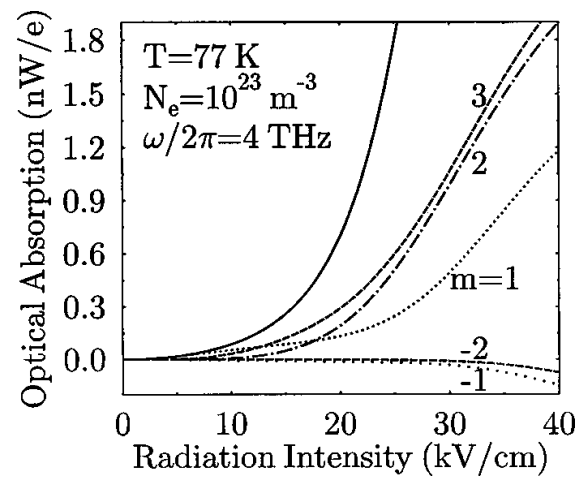

FIG. 7. Optical absorption energy per electron, induced by different photon processes, as a function of radiation intensity at a fixed radiation frequency. The solid curve is the total contributions from all optical processes (see Fig. 4).

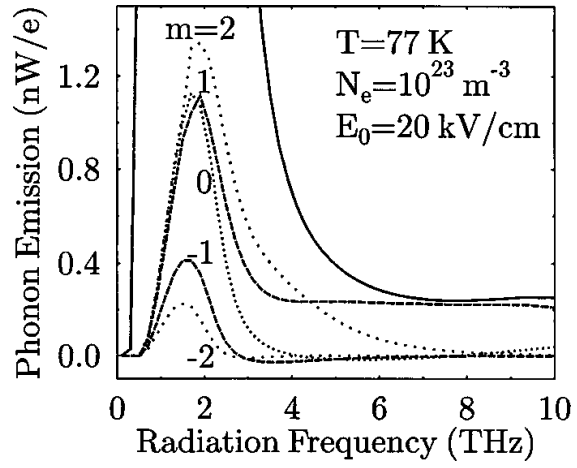

FIG. 8. Intensity of LO-phonon emission, induced by different photon processes, as a function of radiation frequency $\omega / 2 \pi$ at a fixed radiation intensity. $m>0 \quad(m<0)$ corresponds to a $m$-photon absorption (emission) process and the solid curve is the total contributions from all optical channels (see Fig. 3).

From Fig. 7, we note that at a fixed radiation frequency, (i) at low-intensity radiations, optical absorption results mainly from one-photon absorption process (i.e., from $m=1$ ); (ii) the contributions from $m \geqslant 2$ channels and from photon emission to optical absorption increase rapidly with $E_{0}$; and (iii) at high-intensity radiations (e.g., $E_{0}>15 \mathrm{kV} / \mathrm{cm}$ when $\omega / 2 \pi=4 \mathrm{THz}$ ), optical absorption is determined by multiphoton absorption processes (i.e., by those with $m \geqslant 2$ ). From Figs. 6 and 7, one can find that the contribution due to photon emission (i.e., those associated with $m<0$ ) to EELR (or optical absorption) is much smaller than that from photon absorption (i.e., those with $m>0$ ). These theoretical results indicated that multiphoton absorption processes can become the principal channels for optical absorption in bulk semiconductor materials subjected to high-intensity and/or lowfrequency radiations. The radiations provided by FEL's, with $E_{0} \sim 1 \mathrm{kV} / \mathrm{cm}$ and $\omega \sim 1 \mathrm{THz}$, can be used to observe these multiphoton effects. Experimentally, the total energy of optical absorption in an electronic device can be measured through $^{7} \sigma_{x x} E_{0}^{2} / 2=P_{\mathrm{OP}}$, which can be deduced from the data of the applied radiation field and of the conductivity measured at the corresponding radiation.

In the presence of an intense EM field, the emission of LO phonons from an electron gas can be generated via electronic transitions accompanied by the absorption and emission of

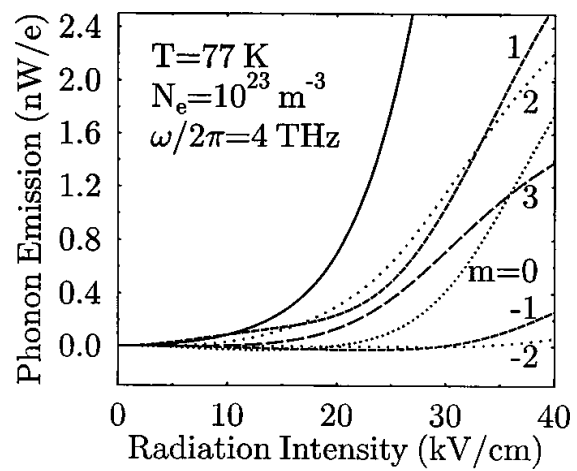

FIG. 9. Intensity of LO-phonon emission, induced by different photon processes, as a function of radiation intensity at a fixed radiation frequency. The solid curve is the total contributions from all optical absorption processes (see Fig. 4). 

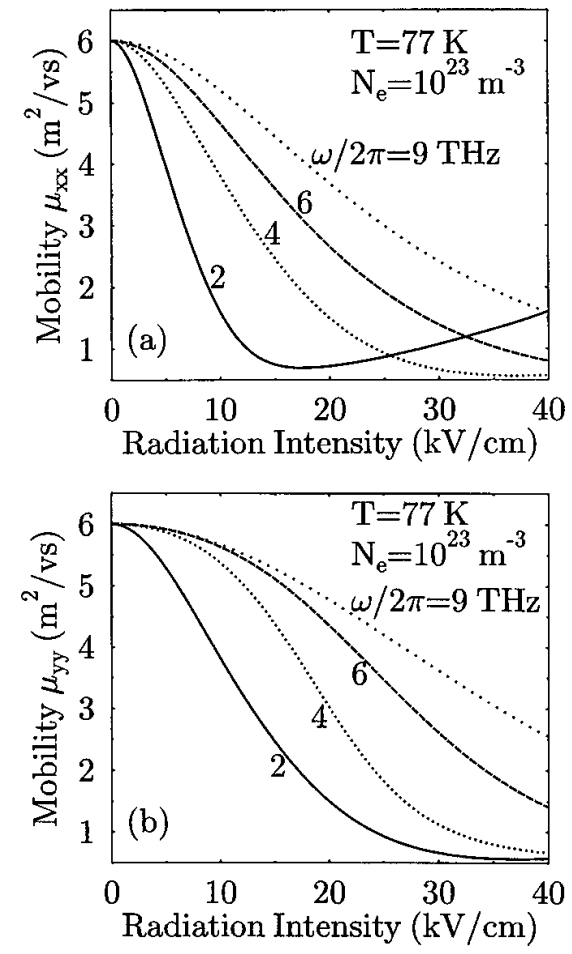

FIG. 10. Mobility, $\mu_{x x}$ in (a) and $\mu_{y y}$ in (b), as a function of radiation intensity for different radiation frequencies. The conductivity $\sigma_{j j}=N_{e} e \mu_{j j}$.

the photons. The contributions from different optical processes to LO-phonon emission in a GaAs-based 3DEG are presented in Figs. 8 and 9. For an electron gas subjected to EM radiations, zero-photon process may contribute to the emission of the phonons. We find that similar to the case of optical absorption via multiphoton processes, LO-phonon emission from a THz-driven 3DEG can be achieved through multiphoton absorption and emission. This effect is more pronounced for radiations with relatively low frequency (see Fig. 8) and/or high intensity (see Fig. 9). The peaks of LOphonon emission via different optical channels can be observed at about $\omega / 2 \pi \sim 2 \mathrm{THz}$ (see Fig. 8). At HF radiations (e.g., $\omega / 2 \pi>6 \mathrm{THz}$ when $E_{0}=20 \mathrm{kV} / \mathrm{cm}$ ), LO-phonon emission results mainly from the one-photon absorption process. In Figs. 8 and 9, the EELR induced by interaction with LO phonons via photon emission $(m<0)$ may be negative. A negative EELR means that the electrons in the system can gain the energy from the corresponding electronic transitions. Together with those observed and discussed for the influence of the radiation field on electron temperature (see Figs. 1 and 2), we find that a negative EELR induced by LO-phonon absorption mediated by photon emission takes main responsibility for the cooling of electron temperature shown in Figs. 1 and 2. When $T_{e}<T$, the rate of LO-phonon absorption scattering via $m<0$ channels is larger than that of emission scattering via $m>0$ channels and, consequently, the EELR caused by LO-phonon scattering becomes negative. It can be seen from Figs. 8 and 9 that for HF and high-intensity radiations, the processes of photon emission affect weakly the LO-phonon emission. The LO-phonon emission is mainly generated by electronic transitions accompanied by the processes of photon absorption.
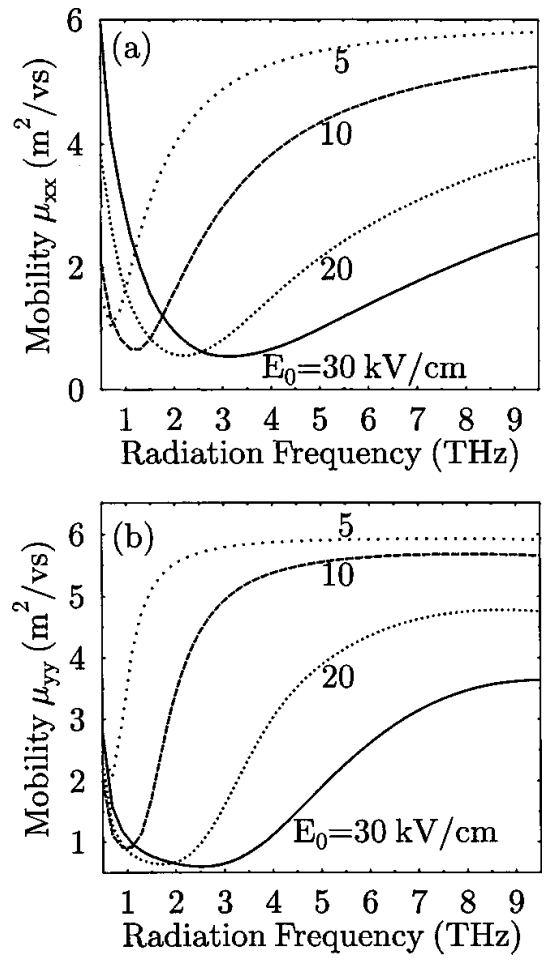

FIG. 11. Mobility, $\mu_{x x}$ in (a) and $\mu_{y y}$ in (b), as a function of radiation frequency $\omega / 2 \pi$ for different radiation intensities.

\section{Mobility and relaxation time}

The dependence of the mobility (or conductivity $\sigma_{j j}$ $=N_{e} e \mu_{j j}$ ) measured in different geometries on the THz EM radiation is shown in Figs. 10-13. When the radiation is polarized along the $x$ direction, $\mu_{i j}$ can be obtained experimentally by applying the dc probing electric field (or current) along the $i$ direction and measuring the current (or electric field) along the $j$ direction. Our theoretical results show that for electron interactions with photons and with LO phonons, $\mu_{x y}=\mu_{y x}=0$ and, normally, $\mu_{x x} \neq \mu_{y y}$, which is evident by Figs. 12 and 13. In Fig. 10, at HF radiations both $\mu_{x x}$ and $\mu_{y y}$ decrease with increasing $E_{0}$. However, a relatively LF radiation (e.g., $\omega / 2 \pi=2 \mathrm{THz}$ ) may result in an increase of $\mu_{x x}$ with $E_{0}$ in the high radiation intensity regime. At a fixed radiation intensity, in Fig. 11 both $\mu_{x x}$ and $\mu_{y y}$ first decrease and then increase with increasing $\omega$. With further increasing $\omega$ (e.g., $\omega / 2 \pi>4 \mathrm{THz}$ at $E_{0}=5 \mathrm{kV} / \mathrm{cm}$ ), the mobilities de-

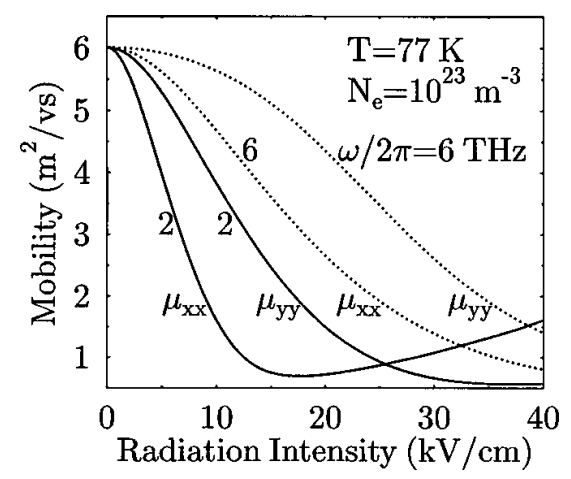

FIG. 12. Different dependence of $\mu_{x x}$ and $\mu_{y y}$ on the radiation intensity is evident. 


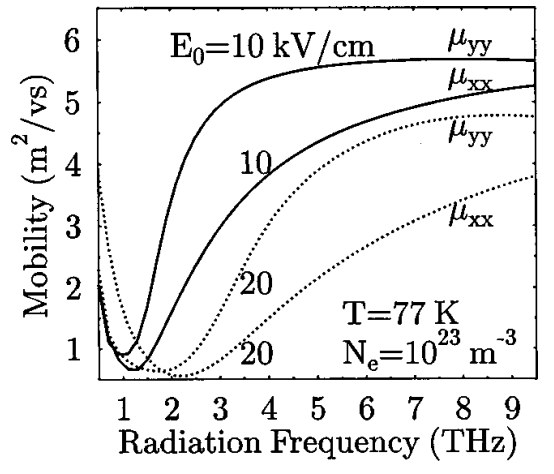

FIG. 13. Different dependence of $\mu_{x x}$ and $\mu_{y y}$ on the radiation frequency is evident.

pend weakly on $\omega$. At a fixed $E_{0}, r_{0} \sim \omega^{-2}$ decreases rapidly with increasing $\omega$. When $r_{0} q_{x} \ll 1$, the electron relaxation time and the EELR depend very little on the EM radiation. From theoretical results given by Eqs. (20) and (23) and shown in Figs. 12 and 13, we can find that the difference between $\mu_{x x}$ and $\mu_{y y}$ can be observed more markedly at a radiation field with higher intensity (see Fig. 12) and intermediate frequency (see Fig. 13). When $E_{0}=0, \mu_{x x}=\mu_{y y}$ due to $\lim _{r_{0} \rightarrow 0} J_{m}^{2}\left(r_{0} q_{x}\right)=\delta_{0, m}$. In the presence of the EM radiations, an anisotropic conduction caused by electron-photonimpurity scattering in semiconductor Si-based structures at low temperatures was predicted by Brandes. ${ }^{17}$ The results presented in this paper indicate that the anisotropic conductivity tensors can also be measured in GaAs-based structures subjected to intense $\mathrm{THz}$ radiations, due to electron-photonphonon interactions in relatively high temperatures.

The dependence of relaxation time (or scattering rate) in different geometries of a THz-driven 3DEG on intensity and frequency of the EM radiations is presented in Figs. 14 and 15. At a fixed radiation intensity and in the LF (HF) regime (see Fig. 14), the electronic scattering is mainly through multiphoton (zero-photon) channels. At a fixed radiation frequency and in the low-intensity (high-intensity) regime (see Fig. 15), zero-photon (multiphoton) process accompanied by the LO-phonon scattering is the main channel for electronic transitions. An important conclusion we draw from those shown in Figs. 14 and 15 is that the rate of electron-photonphonon scattering in a GaAs-based 3DEG structure can be comparable to the frequency of the $\mathrm{THz}$ radiations. This feature implies that $\mathrm{THz}$ radiations may couple strongly to the electronic system and may modify significantly the processes of momentum and energy relaxation for excited electrons in the device. Hence, THz-driven 3DEG's can exhibit some important and distinctive features in their nonlinear transport and optical properties.

\section{The case of low-frequency radiations}

The results discussed and shown above are mainly for the case of $\omega / 2 \pi \sim 1 \mathrm{THz}$. Now we discuss some features obtained from the present theory for 3DEG's subjected to lowfrequency (i.e., $\omega \ll 1$ and $\omega \rightarrow 0$ ) EM radiations. Theoretical results given by Eq. (24c) and shown in Fig. 3 indicate that in the presence of the weak probing fields and when the radiation frequency $\omega \rightarrow 0, P_{\mathrm{OP}} \sim \hbar \omega \rightarrow 0$, which leads to
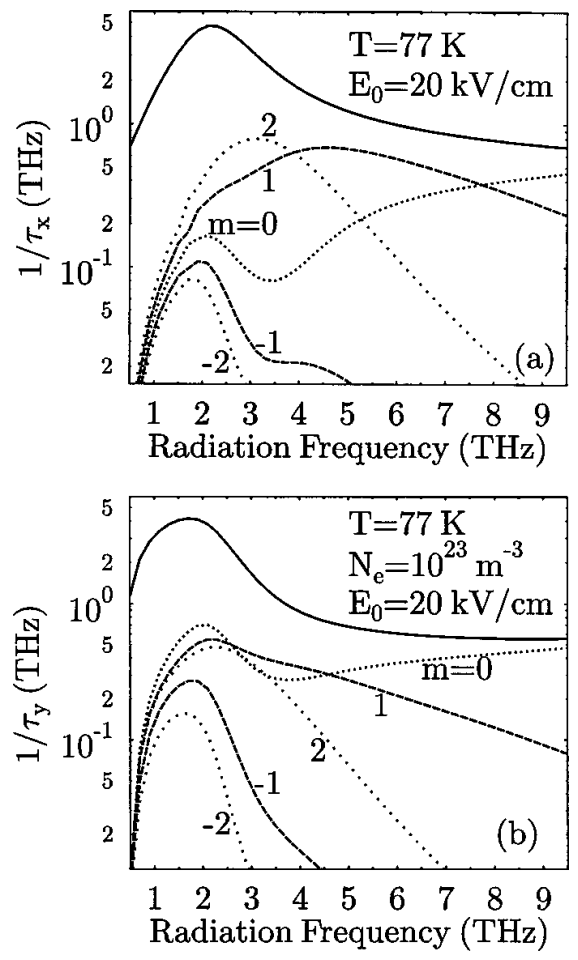

FIG. 14. Scattering rate, $1 / \tau_{x}$ in (a) and $1 / \tau_{y}$ in (b) induced by different photon processes, as a function of radiation frequency $\omega / 2 \pi$ at a fixed radiation intensity. $m>0(m<0)$ corresponds to a $m$-photon absorption (emission) process and the solid curve is the total contributions from all optical channels.

$T_{e} \rightarrow T$ (see Fig. 1) for all radiation intensities. For LF radiations, the photon energy $\hbar \omega$ is very small. In this case, electron interactions with the radiation fields via photon emission and absorption will be less efficient. In the present study, the EM radiation has been taken into account within the Cou-

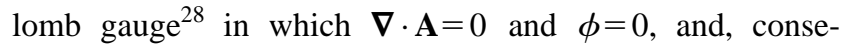
quently, $\rho=0$ and $\mathbf{j}=0$ for free electrons. The $\omega \rightarrow 0$ limit of this approach is essentially different from those by simply taking the radiation field as an ac electric field, which corresponds to a gauge variation: $\mathbf{A} \rightarrow 0$ and $\phi \rightarrow E_{0} x \cos (\omega t)$. When an EM radiation is taken as an ac electric field, we have the following. (1) $\rho=0$ and $\mathbf{j}=0$ cannot be satisfied. Therefore, it can only be used for the case of interacting electrons through, e.g., taking into consideration in the lefthand side of the Boltzmann equation. ${ }^{7}$ (2) The solution of the Schrödinger equation at the $\omega \rightarrow 0$ limit is characterized by the Airy functions, which will result in different electronic transition rate after using the time-dependent perturbation theory. (3) A gauge-translational approach ${ }^{29}$ has to be employed in order to study, e.g., the transport problem. However, by using the coordinate transformation proposed by Truscott, ${ }^{30}$ the time-dependent Schrödinger equation in which the radiation field is taken as an ac electric field for $\omega \neq 0$ can also be solved analytically. It can be justified that the electronic transition rate obtained from using this wave function is the same as those given by Eqs. (4) and (5). This implies that Eqs. (4) and (5) are gauge invariant. So, the only problem that arises from taking the EM radiation field as a vector potential alone or as an ac electric field alone is at the 

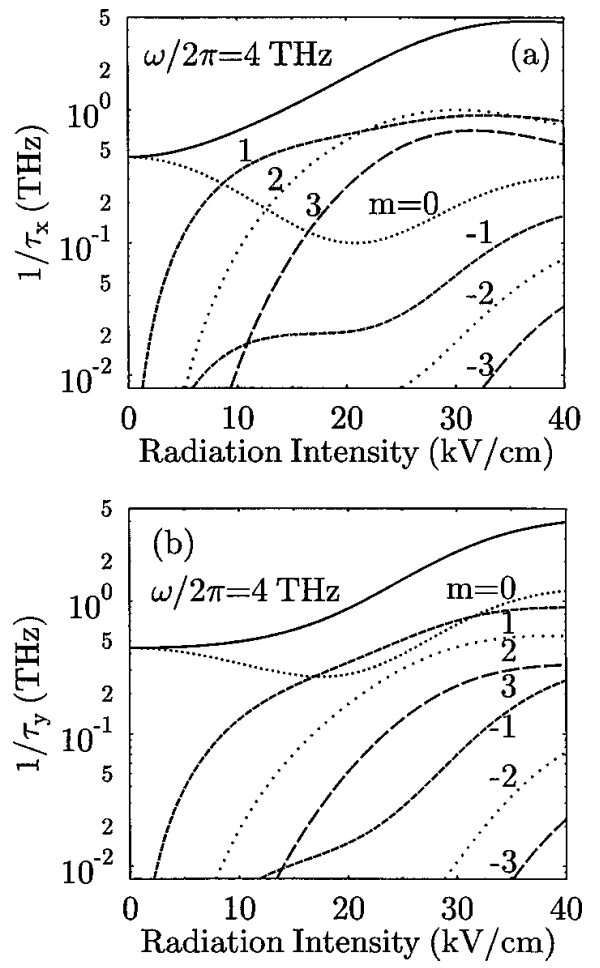

FIG. 15. Scattering rate, $1 / \tau_{x}$ in (a) and $1 / \tau_{y}$ in (b) induced by different photon processes, as a function of radiation intensity at a fixed radiation frequency. The solid curve is the total contributions from all optical channels.

$\omega \rightarrow 0$ limit. The theoretical results shown in this paper indicate that at the $\omega \rightarrow 0$ limit, an EM radiation field cannot be treated as an ac electric field.

For the case of a radiation field with low frequency and low intensity so that $\omega \ll \omega_{\mathrm{LO}}$ and $r_{0} q_{x} \sim 1$, due to $\sum_{m=-\infty}^{\infty} J_{m}^{2}(x)=1$ in Eq. (23), the momentum-relaxation time is independent of $\omega$ and $E_{0}$ and can be determined by Eq. (25a). The mobility now is given by $\mu_{x x}=\mu_{y y}=e \tau / m^{*}$. For the case of a radiation field with low frequency and high intensity (noting $r_{0} \sim E_{0} / \omega^{2}$ ) so that $r_{0} q_{x} \gg m$, the relaxation time in different geometries [see Eq. (23)] and the EELR [see Eqs. (24)] may depend on the intensity and frequency of the radiation field, because when $x \gg m, J_{m}(x) \sim x^{-1 / 2} \cos (x$ $-m \pi / 2-\pi / 4)$.

In the calculations, we only include the contributions from optical processes regarding $m=0, \pm 1, \pm 2, \ldots$, and \pm 20 , to save CPU time. From the fact that a stronger effect of electron-photon-phonon interactions can be observed when $|m| \omega \sim \omega_{\mathrm{LO}}$, in LF regime, more multiphoton processes have to be included. It should be pointed out that in Figs. 11, 13, and 14, we have shown the results for the case of $\omega / 2 \pi>0.5 \mathrm{THz}$ where a high accuracy of the numerical calculation can be achieved. When $\omega / 2 \pi<0.5 \mathrm{THz}$, the mobility (inverse relaxation time) induced by LO-phonon scattering increases (decreases) monotonically with decreasing $\omega$, because $\omega \ll \omega_{\text {LO }}$ so that the electron-LO-phonon interaction via photon emission and absorption becomes weaker. Under the very LF radiations, the electronic transitions can be achieved via other scattering mechanisms, such as acoustic phonons and impurities that were not taken into consideration in the present study. For a GaAs-based electron gas system, the frequency of the acoustic phonons is at about $\omega_{Q} / 2 \pi \simeq 0.1 \mathrm{THz},{ }^{31}$ so one would expect that in the presence of the LF EM radiations with $\omega / 2 \pi \sim 0.1 \mathrm{THz}$ the electronacoustic-phonon interaction will be an important channel for relaxation of excited electrons. Furthermore, the impurityinduced optical absorption in a 3DEG device occurs in relatively LF regime and depends strongly on sample parameters.

The LF and $\omega \rightarrow 0$ conductivity was discussed in Ref. 24 for electron-LO-phonon interactions. In Ref. 24, to calculate correctly the LF (especially as $\omega \rightarrow 0$ ) conductivity, one has to sum an infinite number of diagrams. In contrast, using the approaches developed in the present study, we can calculate the LF conductivity by summing over an infinite number of $m$, which is much easier to do.

\section{SUMMARY}

In this paper, we have performed a detailed theoretical study of the nonlinear transport and optical properties in THz-driven 3DEG's. We have developed an approach to deal with electron-photon-phonon interactions in a $3 \mathrm{DEG}$ structure. Using this approach, the effect of the EM radiation can be considered more exactly and the effect of multiphoton processes on the transport and optical properties in an electron gas can be more easily and directly included. Introducing the electronic transition rate induced by electron-photonphonon interactions into the steady-state Boltzmann equation, we have derived the momentum- and energybalance equations, which can be used to study the nonlinear transport and optical effects measured by, e.g., transport experiments. These theoretical approaches have been employed to study the dependence of electron temperature, relaxation time, mobility, multiphoton absorption and emission, and photon-assisted phonon emission on frequency and intensity of the $\mathrm{THz}$ radiations in a GaAs-based 3DEG structure. The main results obtained from this study are summarized as follows.

For a THz-driven 3DEG, at relatively low-frequency and low-intensity radiations, the electrons in the system will be slightly cooling down, which results from predominantly forward scattering induced by the LO-phonon scattering mechanism. The cooling of electrons in the device system will result in a stronger LO-phonon absorption scattering via channels of photon emission and, consequently, in the energy gain achieved by phonon scattering via the corresponding optical processes. The heating of electrons can be observed more markedly at a radiation with relatively lower frequency and higher intensity.

At a fixed radiation intensity, a peak in the optical absorption and/or phonon emission can be observed for a 3DEG system at about $\omega / 2 \pi \sim 1 \mathrm{THz}$. With increasing radiation intensity, the peak shifts to the high-frequency regime and looks more broadened. These features are very similar to the resonant absorption observed in THz-driven 2DEG's where, however, the spectrum of $\mathrm{THz}$ optical absorption was shown experimentally ${ }^{1}$ at a fixed electron temperature and the peak was observed at about $\omega / 2 \pi \sim 0.5 \mathrm{THz}$.

For a THz-driven 3DEG, low-frequency and/or highintensity optical absorption can be achieved via multiphoton processes. At a relatively high-frequency and/or low- 
intensity radiation, the process of one-photon absorption is a principal channel for optical absorption. The electronphoton-phonon interactions via channels of photon emission affect weakly the electron mobility and the EELR. The photon absorption and emission via electron-photon-phonon interactions is an indirect optical mechanism. The results show that GaAs-based 3DEG structures can be used as nonlinear electronic and optical devices working at FIR or $\mathrm{THz}$ regime.

In the presence of an intense EM field, the emission of LO phonons from an electron gas can be generated via electronic transitions accompanied by the absorption of photons. LOphonon emission via multiphoton absorption can be achieved at radiations with relatively low frequencies and/or high intensities.

The presence of an EM radiation polarized along a certain direction will break the symmetry of a 3DEG structure. As a consequence, (1) an anisotropic nature of the electronic transition rate can be observed for electron-photon-phonon interactions; (2) the momentum-relaxation time in different geometries will be different; and (3) the anisotropic conductivity tensors can be measured. A larger difference between $\sigma_{x x}$ and $\sigma_{y y}$ can be observed at a radiation field with higher intensity and intermediate frequency.
The rate of electron-photon-phonon scattering in a GaAsbased 3DEG structure can be comparable to the frequency of the $\mathrm{THz}$ radiations, through varying the intensity and/or frequency of the radiation field. It implies that $\mathrm{THz}$ radiations may couple strongly to the electronic system and may modify significantly the processes of momentum and energy relaxation for excited electrons in the device. And this is the main reason why some important and distinctive features in the nonlinear transport and optical properties can be observed in THz-driven 3DEG's.

Finally, the phenomena discussed and predicated in the present study may be observed within the radiation intensity and frequency regimes of recently developed free-electron lasers such as UCSB (Refs. 1-3) FEL's and FELIX. ${ }^{4,5}$ We hope those presented in this paper could be verified experimentally.

\section{ACKNOWLEDGMENTS}

This work was supported by the Australian Research Council. The author is grateful to S. M. Stewart for his reading of the manuscript.
*Electronic address: wxu@wumpus.its.uow.edu.au

${ }^{1}$ N. G. Asmar, A. G. Markelz, E. G. Gwinn, J. Cerne, M. S. Sherwin, K. L. Campman, P. F. Hopkins, and A. C. Gossard, Phys. Rev. B 51, 18041 (1995).

${ }^{2}$ N. G. Asmar, J. Cerne, A. G. Markelz, E. G. Gwinn, M. S. Sherwin, K. L. Campman, and A. C. Gossard, Appl. Phys. Lett. 68, 829 (1996).

${ }^{3}$ A. G. Markelz, N. G. Asmar, B. Brar, and E. G. Gwinn, Appl. Phys. Lett. 69, 3975 (1996).

${ }^{4}$ B. N. Murdin, W. Heiss, C. J. G. M. Langerak, S.-C. Lee, I Galbraith, G. Strasser, E. Gornik, M. Helm, and C. R. Pidgeon, Phys. Rev. B 55, 5171 (1997).

${ }^{5}$ See, e.g., C. J. G. M. Langerak, B. N. Murdin, B. E. Cole, J. M. Chamberlain, M. Henini, M. Pate, and G. Hill, Appl. Phys. Lett. 67, 3453 (1995).

${ }^{6}$ W. Xu and C. Zhang, Appl. Phys. Lett. 68, 3305 (1996).

${ }^{7}$ W. Xu and C. Zhang, Phys. Rev. B 55, 5259 (1997).

${ }^{8}$ W. Xu and C. Zhang, Phys. Rev. B 54, 4907 (1996).

${ }^{9}$ N. Tzoar, P. M. Platzman, and A. Simons, Phys. Rev. Lett. 36, 1200 (1976), and references for their early work up to 1963 therein.

${ }^{10}$ W. Götze and P. Wölfle, Phys. Rev. B 6, 1226 (1972).

${ }^{11}$ Z. Q. Zou and X. L. Lei, Phys. Rev. B 51, 9493 (1995), and references for the early work by X. L. Lei et al. therein.

${ }^{12}$ S. K. Ma and K. W.-K. Shung, Phys. Rev. B 48, 10751 (1993).

${ }^{13}$ W. Cai, P. Hu, T. F. Zheng, B. Yudanin, and M. Lax, Phys. Rev. B 40, 7671 (1989), and references for their early work therein.

${ }^{14}$ X. L. Lei and N. J. M. Horing, Phys. Rev. B 36, 4238 (1987).

${ }^{15}$ N. Tzoar, Phys. Rev. 132, 202 (1963).
${ }^{16}$ A. P. Jauho and K. Johnsen, Phys. Rev. Lett. 76, 4576 (1996); W. Xu, J. Phys.: Condens. Matter 9, L591 (1997); Semicond. Sci. Technol. 12, 1559 (1997).

${ }^{17}$ T. Brandes, Europhys. Lett. 33, 629 (1996).

${ }^{18}$ See, e.g., A. Messiah, Quantum Mechanics (North-Holland, Amsterdam, 1970), Vol. II

${ }^{19}$ For a review, see, e.g., K. Seeger, Semiconductor Physics-An Introduction (Springer-Verlag, Berlin, 1991).

${ }^{20}$ See, e.g., H. Y. Fan, W. Spitzer, and R. J. Collins, Phys. Rev. 101, 566 (1956)

${ }^{21}$ W. Xu, F. M. Peeters, and J. T. Devreese, Phys. Rev. B 43, 14134 (1991).

${ }^{22}$ See, e.g., D. Chattopadhyay and H. J. Queisser, Rev. Mod. Phys. 53, 745 (1981).

${ }^{23}$ For a review, see, e.g., J. S. Blakemore, J. Appl. Phys. 53, R123 (1982).

${ }^{24}$ For a review, see, e.g., F. M. Peeters and J. T. Devreese, Solid State Phys. 30, 81 (1984).

${ }^{25}$ See, e.g., E. M. Conwell, High Field Transport in Semiconductors, Solid State Physics Supplement 9 (Academic, New York, 1967).

${ }^{26}$ W. Xu, F. M. Peeters, and J. T. Devreese, Phys. Rev. B 46, 7571 (1992).

${ }^{27}$ See, e.g., C. H. Yang, J. M. Carlson-Swindle, S. A. Lyon, and J. M. Worlock, Phys. Rev. Lett. 55, 2359 (1985).

${ }^{28}$ See, e.g., R. Shankar, Principles of Quantum Mechanics (Plenum, New York, 1980).

${ }^{29}$ See, e.g., N. Pottier and D. Calecki, Physica A 110, 471 (1982).

${ }^{30}$ W. S. Truscott, Phys. Rev. Lett. 70, 1900 (1993).

${ }^{31}$ See, e.g., W. Xu, Appl. Phys. Lett. 68, 1353 (1996) 